\title{
Comunidade de Coleoptera de solo em remanescente de Mata Atlântica no Estado do Rio de Janeiro, Brasil
}

\author{
Cíntia Cristina Lima Teixeira ${ }^{1}$, Magali Hoffmann ${ }^{2}$ \& Gilson Silva-Filho ${ }^{2}$ \\ ${ }^{1}$ Laboratório de Zoologia, Departamento de Biologia, Centro Universitário São Camilo-ES - CUSC-ES, \\ Rua São Camilo de Léllis, 01, Bairro Paraíso, CEP 29304-910, Cachoeiro de Itapemirim, ES, Brazil \\ e-mail: cintiateixeira@sãocamilo-es.br \\ ${ }^{2}$ Laboratório de Entomologia e Fitopatologia - LEF, Centro de Ciências e Tecnologias Agropecuárias - CCTA, \\ Universidade Estadual do Norte Fluminense Darcy Ribeiro - UENF, \\ Av. Alberto Lamego, 2000, CEP 28013-600, Campos dos Goytacazes, RJ, Brazil \\ e-mail:magali@uenf.br; gilson@uenf.br
}

TEIXEIRA, C.C.L., HOFFMANN, M. \& SILVA-FILHO, G. Comunity of soil fauna Coleoptera in the remnants of lowland Atlantic Forest in state of Rio de Janeiro, Brazil. Biota Neotrop., 9(4): http://www.biotaneotropica. org.br/v9n4/en/abstract?article+bn02709042009.

\begin{abstract}
The soil fauna plays an important role in the ecological function of an ecosystem and studies on the composition and structure of these communities are important to understand your functioning. The objective of our study was to evaluate of community of the soil fauna Coleoptera, collected bimonthly through pitfall trap, from August 2003 to August 2004. A total of 10,820 specimens from 24 families of Coleoptera were captured, but Nitidulidae (4,782 individuals), Curculionidae (3,176), Scarabaeidae (2,019) and Staphylinidae (326) were considered abundant families. According to the trophic guild, the collected individuals can be divided into detritivores $(4,802$ specimens), herbivores $(3,218)$, decomposers $(2,019)$, predators $(621)$ and fungivoros (160). These results indicate no dominance of a single trophic group, however, the high occurrence of detritivores (44.4\%), herbivores (29.7\%) and decomposers (18.7\%) was registered. About 61.8\% (6,685 individuals) were captured during October 2003 to March 2004, a rainy period, and 38.2\% (4,135) during the dry period indicating a pronounced seasonal difference in the relative abundance of individuals sampled. The high frequency of individuals was observed during the October and December, 2003, and March, 2004.
\end{abstract}

Keywords: pitfall traps, trophic guild, detritivores, decomposers, herbivores.

TEIXEIRA, C.C.L., HOFFMANN, M. \& SILVA-FILHO, G. Comunidade de Coleoptera de solo em remanescente de Mata Atlântica no estado do Rio de Janeiro, Brasil. Biota Neotrop., 9(4): http://www. biotaneotropica.org.br/v9n4/pt/abstract?article+bn02709042009.

Resumo: A fauna do solo exerce importante papel nos processos ecológicos do ecossistema. Estudos sobre a composição e estrutura dessas comunidades são importantes para entender o seu funcionamento. Assim o objetivo deste trabalho foi avaliar a comunidade de Coleoptera, coletada mediante armadilhas de solo, quinzenalmente, durante o período de treze meses (08/2003-08/2004), na Mata do Mergulhão, Rio de Janeiro. Foram capturados 10.820 espécimes, representados por 24 famílias, sendo considerados abundantes Nitidulidae (4.782 indivíduos), Curculionidae (3.176 indivíduos), Scarabaeidae (2.019 indivíduos) e Staphylinidae (326 indivíduos), capturadas em todo período de coleta. De acordo com as guildas tróficas, os indivíduos das famílias coletados, podem ser agrupados em detritívoros (4.802 espécimes), herbívoros (3.218 espécimes), decompositores (2.019 espécimes), predadores (621 espécimes) e fungívoros (160 espécimes). Foi constatada maior ocorrência de detritívoros $(44,4 \%)$, herbívoros $(29,7 \%)$ e decompositores $(18,7 \%)$. Foram capturados 6.685 exemplares $(61,8 \%)$ no período de outubro/2003 a março/2004, na estação chuvosa, e 4.135 exemplares $(38,2 \%)$ na estação seca. Foram observadas maiores capturas nos meses de outubro e dezembro de 2003, e março de 2004.

Palavras-chave: armadilhas de solo, guilda trófica, detritivoros, decompositores, herbívoros. 


\section{Introdução}

A Mata Atlântica é considerada como um dos mais ricos conjuntos de ecossistemas em termos de diversidade biológica do Planeta (Myers et al. 2000). No Brasil a fragmentação é acentuada e seus efeitos podem inviabilizar sua preservação, por alterar as características primárias e modificar a composição e a riqueza de espécies na comunidade (Schoereder et al. 2004).

Uma forma de avaliar o grau de alteração ou fragmentação de um habitat é mediante a utilização de um grupo de organismos considerados como bioindicadores (Wink et al. 2005). Dentre os vários grupos de animais, os insetos são os mais importantes indicadores, principalmente os da ordem Coleoptera, que têm sido alvo de muitos estudos para avaliação de condições ambientais, devido à variedade de seus hábitos alimentares, nichos ecológicos, riqueza de famílias e diversidade de espécies. Para estas avaliações são comuns o uso de parâmetros, como: número de indivíduos por espécie, número de espécies e número de indivíduos por família. Estes parâmetros de abundância e de riqueza são analisados separadamente ou em conjunto para definir padrões de estrutura da comunidade (Ganho \& Marinoni 2003, Wink et al. 2005).

O estudo da fauna de Coleoptera de solo, envolvendo famílias ou espécies abundantes, tem sido alvo de muitos pesquisadores, como forma de consolidar o grupo como indicador de condições ambientais (Marinoni \& Ganho 2003b). Assim, o objetivo deste estudo foi realizar um inventário da fauna de Coleoptera, a partir dos dados de famílias e indivíduos, capturados com armadilha de solo.

\section{Material e Métodos}

Os insetos foram coletados na Mata do Mergulhão (fragmento de Mata Atlântica de baixada com 18 ha), Fazenda Airizes, localizada no município de Campos dos Goytacazes, Estado do Rio de Janeiro, junto a BR 356, km 7, a $11 \mathrm{~m}$ de altitude a $21^{\circ} 45^{\prime} \mathrm{S}$ e $41^{\circ} 18^{\prime} \mathrm{O}$ (Carvalho et al. 2006). A área de amostragem era composta por duas subáreas ( $\mathrm{A}=8$ ha e $\mathrm{B}=10$ ha), aparentemente similares entre si em relação à cobertura vegetal, separada uma da outra por um corredor de $50 \mathrm{~m}$ de largura, e as duas subáreas no período de coletas eram parcialmente circundadas pelo cultivo de cana-de-açúcar.

A amostragem foi realizada quinzenalmente, por meio de armadilha de solo, no período de agosto de 2003 a agosto de 2004. As armadilhas do tipo "pitfall" eram confeccionadas com potes de vidro com $13 \mathrm{~cm}$ de altura e 7,5 cm de diâmetro e capacidade para $500 \mathrm{~mL}$. Estas eram mantidas enterradas de forma que sua abertura ficasse exatamente ao nível da superfície do solo apenas com líquido conservante (formol a 4\%), sem a utilização de atrativos (Marinoni \& Dutra 1996, Marinoni \& Ganho 2003b).

Foram utilizadas 24 armadilhas de solo, sendo 12 por subáreas, considerando-se a similaridade destas. As armadilhas foram instaladas ao longo de trilhas existentes no interior da mata. Todas as primeiras armadilhas eram mantidas numa distância mínima de 20 m da borda da mata e distanciadas entre si em $10 \mathrm{~m}$, sendo dispostas, na mata, de 20 a $120 \mathrm{~m}$ da borda.

Durante todo o experimento cinco pontos de amostragens foram danificados e perdidos.

Os coleópteros capturados foram montados em alfinetes entomológicos, etiquetados e separados em morfo-espécie. Posteriormente foram identificados em nível de família conforme a classificação proposta por Lawrence et al. (1999).

O material etiquetado foi depositado no Museu de Entomologia do Laboratório de Entomologia e Fitopatologia (LEF), do Centro de Ciências e Tecnologias Agropecuárias (CCTA), da Universidade Estadual do Norte Fluminense Darcy Ribeiro (UENF).
A diversidade de espécies e equitabilidade da fauna de Coleoptera foram avaliadas com auxílio dos índices de diversidade de ShannonWiener (H') e Simpson calculados com Log na base "e", segundo Magurran (1988).

As médias do número de coleópteros capturados no período de agosto de 2003 a agosto de 2004 para as duas subáreas foram comparadas pela análise de variância (ANOVA) com auxílio do programa SAS versão 2000.

As análises dos dados sazonais foram baseadas no número total de insetos coletados em cada mês. Estes números foram avaliados junto aos valores médios mensais das variáveis meteorológicas (temperatura média, precipitação e umidade relativa do ar). Os dados metereológicos foram adquiridos da Estação Evapotranspirométrica da PESAGRO-RJ, Laboratório de Engenharia Agrícola da Universidade Estadual do Norte Fluminense Darcy Ribeiro (LEAG/UENF).

\section{Resultados e Discussão}

Foram coletados 10.820 espécimes pertencentes a 24 famílias (Tabela 1). As famílias capturadas em maior quantidade foram Nitidulidae (4.782 indivíduos), Curculionidae (3.176 indivíduos), Scarabaeidae (2.019 indivíduos) e Staphylinidade (326 indivíduos), enquanto as coletadas em menor quantidade foram Aderidae, Anthicidae, Anthribidae, Cerambycidae, Chelonariidae, Endomychidae, Lampyridae, Meloidae e Silvanidae, com apenas um espécime coletado.

Do total de espécimes capturados, 95,3\% (10.312 espécimes) pertencem às quatro famílias mais numerosas, Nitidulidae, Curculionidae, Scarabaeidae e Staphylinidae que podem ser consideradas como abundantes por terem sido capturadas em todos os períodos de coleta nas armadilhas (Tabela 2). Resultados similares foram constatados por Marinoni \& Ganho (2003b) e Petroni (2008), onde a dominância foi atribuida, geralmente, a três ou quatro famílias. Segundo Schubart \& Beck (1968), Rodrigues (1992), Carlton \& Robison (1998) e Chung et al. (2000), são consideradas famílias mais abundantes as que constituem cerca de $85 \%$ do total de indivíduos coletados, no entanto Didham et al. (1998 a, b). Consideram que as famílias mais abundantes constituíram cerca de $66 \%$ do total de espécimes coletados.

Os indivíduos coletados nos 10 diferentes intervalos de distribuição das armadilhas, entre 20 a $120 \mathrm{~m}$ da borda da mata, apresentaram percentuais similares de ocorrência por distância exceto na distância de 80 a 90 m (Tabela 2). Resultados contrastantes foram observados por Marinoni \& Ganho (2003b), estes autores constataram maior abundância no interior da floresta. No entanto, Didham et al. (1998a, b) verificaram que o aumento da população era em direção à borda da mata.

Foi constatada na mata do Mergulhão que as famílias mais abundantes, embora coletadas em todas as distâncias, apresentaram um padrão de dominância, durante todo experimento, nas seguintes distâncias, em relação à borda da mata: Nitidulidae $(55,9 \%)$ de 100 a $110 \mathrm{~m}$, Curculionidae $(42,4 \%)$ de 50 a $60 \mathrm{~m}$, Scarabaeidae $(35,6 \%)$ de 70 a 80 m e Staphylinidae $(6,9 \%)$ de 40 a 50 m (Tabela 2).

As famílias Corylophidae (1,0\%), Latridiidae $(0,5 \%)$ e Scydmaenidae $(0,5 \%)$, mesmo não sendo consideradas abundantes, foram coletadas, em pelo menos, cinco das distâncias entre 20 e 120 m da borda. Corylophidae foi coletada em, praticamente, todas as distâncias exceto, de 90 a 100 m, enquanto Latridiidae e Scydmaenidae foram capturadas em, apenas, cinco distâncias e nunca as duas na mesma distância. Isto, provavelmente, indica que Corylophidae habita tanto as áreas de borda quanto de interior, no entanto indivíduos das famílias Latridiidae e Scydmaenidae não coabitam os mesmos locais (Tabela 2). 
Tabela 1. Relação de famílias e quantidade de Coleoptera capturadas mensalmente em armadilhas de agosto 2003 a agosto de 2004 na mata do Mergulhão em Campos dos Goytacazes, RJ.

Table1. Relation of families and captured amount of the Coleoptera monthly in pitfall trap during August of 2003 to August of 2004 in the remnants of lowland Atlantic Forest in Campos dos Goytacazes, RJ.

\begin{tabular}{|c|c|c|c|c|c|c|c|c|c|c|c|c|c|c|}
\hline \multirow[t]{3}{*}{ Famílias } & \multicolumn{13}{|c|}{ Período } & \multirow[t]{3}{*}{ Total } \\
\hline & \multicolumn{5}{|c|}{2003} & \multicolumn{8}{|c|}{2004} & \\
\hline & Ago. & Set. & Out. & Nov. & Dez. & Jan. & Fev. & Mar. & Abr. & Maio & Jun. & Jul. & Ago. & \\
\hline Nitidulidae & 196 & 231 & 636 & 462 & 1009 & 366 & 456 & 459 & 295 & 166 & 146 & 110 & 250 & 4782 \\
\hline Curculionidae & 23 & 56 & 92 & 141 & 336 & 166 & 279 & 359 & 337 & 345 & 551 & 231 & 260 & 3176 \\
\hline Scarabaeidae & 203 & 21 & 382 & 205 & 429 & 177 & 61 & 156 & 179 & 109 & 68 & 14 & 15 & 2019 \\
\hline Staphylinidae & 7 & 11 & 13 & 5 & 68 & 21 & 50 & 13 & 1 & 6 & 42 & 19 & 70 & 326 \\
\hline Carabidae & 0 & 7 & 29 & 58 & 54 & 12 & 19 & 12 & 8 & 5 & 0 & 0 & 0 & 204 \\
\hline Mordellidae & 0 & 0 & 1 & 0 & 0 & 2 & 0 & 1 & 0 & 0 & 0 & 0 & 0 & 4 \\
\hline Meloidae & 0 & 0 & 0 & 1 & 0 & 0 & 0 & 0 & 0 & 0 & 0 & 0 & 0 & 1 \\
\hline Lampyridae & 0 & 0 & 1 & 0 & 0 & 0 & 0 & 0 & 0 & 0 & 0 & 0 & 0 & 1 \\
\hline Histeridae & 1 & 0 & 3 & 3 & 1 & 3 & 2 & 0 & 1 & 0 & 0 & 0 & 2 & 16 \\
\hline Endomychidae & 0 & 0 & 0 & 1 & 0 & 0 & 0 & 0 & 0 & 0 & 0 & 0 & 0 & 1 \\
\hline Elateridae & 0 & 0 & 1 & 3 & 1 & 1 & 1 & 0 & 0 & 0 & 0 & 0 & 0 & 7 \\
\hline Chrysomelidae & 0 & 0 & 0 & 3 & 7 & 2 & 0 & 1 & 1 & 0 & 2 & 0 & 0 & 16 \\
\hline Cerambycidae & 0 & 0 & 0 & 0 & 0 & 0 & 1 & 0 & 0 & 0 & 0 & 0 & 0 & 1 \\
\hline Tenebrionidae & 0 & 0 & 1 & 2 & 3 & 0 & 1 & 3 & 2 & 0 & 4 & 1 & 2 & 19 \\
\hline Anthribidae & 0 & 0 & 0 & 1 & 0 & 0 & 0 & 0 & 0 & 0 & 0 & 0 & 0 & 1 \\
\hline Anobiidae & 0 & 0 & 2 & 2 & 5 & 0 & 0 & 0 & 0 & 0 & 2 & 0 & 0 & 11 \\
\hline Corylophidae & 20 & 35 & 24 & 22 & 4 & 0 & 0 & 1 & 0 & 0 & 0 & 0 & 0 & 106 \\
\hline Latridiidae & 4 & 3 & 5 & 0 & 1 & 0 & 1 & 0 & 0 & 0 & 11 & 4 & 24 & 53 \\
\hline Scydmaenidae & 1 & 12 & 6 & 7 & 6 & 2 & 2 & 1 & 1 & 1 & 8 & 2 & 3 & 52 \\
\hline Chelonariidae & 0 & 0 & 0 & 0 & 0 & 0 & 0 & 0 & 1 & 0 & 0 & 0 & 0 & 1 \\
\hline Anthicidae & 0 & 1 & 0 & 0 & 0 & 0 & 0 & 0 & 0 & 0 & 0 & 0 & 0 & 1 \\
\hline Silvanidae & 0 & 0 & 1 & 0 & 0 & 0 & 0 & 0 & 0 & 0 & 0 & 0 & 0 & 1 \\
\hline Hydrophilidae & 1 & 0 & 0 & 2 & 9 & 2 & 1 & 2 & 1 & 0 & 2 & 0 & 0 & 20 \\
\hline Aderidae & 0 & 0 & 1 & 0 & 0 & 0 & 0 & 0 & 0 & 0 & 0 & 0 & 0 & 1 \\
\hline Total & 456 & 377 & 1198 & 918 & 1933 & 754 & 874 & 1008 & 827 & 632 & 836 & 381 & 626 & 10820 \\
\hline
\end{tabular}

Tabela 2. Distribuição das famílias de Coleoptera (\%), por distância das armadilhas de solo instaladas entre 20 e 120 m da borda, na Mata do Mergulhão, Campos dos Goytacazes (RJ), de agosto de 2003 a agosto de 2004.

Table 2. Distribution of Coleoptera's families (\%), by distance of the installed pitfall traps between 20 and $120 \mathrm{~m}$ of the edge, in the remnants of lowland Atlantic Forest, during August of 2003 to August of 2004.

\begin{tabular}{|c|c|c|c|c|c|c|c|c|c|}
\hline $\begin{array}{c}\text { 20-30 } \\
\text { Família }\end{array}$ & $\%$ & $\begin{array}{c}30-40 \\
\text { Família }\end{array}$ & $\%$ & $\begin{array}{c}40-50 \\
\text { Família }\end{array}$ & $\%$ & $\begin{array}{c}50-60 \\
\text { Família }\end{array}$ & $\%$ & $\begin{array}{c}60-70 \\
\text { Família }\end{array}$ & $\%$ \\
\hline Nitidulidae & 53,5 & Nitidulidae & 45,2 & Nitidulidae & 53,9 & Curculionidae & 42,4 & Curculionidae & 40,9 \\
\hline Curculionidae & 23,6 & Curculionidae & 27,5 & Scarabaeidae & 25,0 & Nitidulidae & 34,5 & Nitidulidae & 38,6 \\
\hline Scarabaeidae & 12,5 & Scarabaeidae & 21,9 & Curculionidae & 11,5 & Scarabaeidae & 17,2 & Scarabaeidae & 16,1 \\
\hline Staphylinidae & 3,4 & Staphylinidae & 1,9 & Staphylinidae & 6,9 & Staphylinidae & 2,2 & Staphylinidae & 1,7 \\
\hline Carabidae & 2,0 & Carabidae & 1,6 & Carabidae & 1,3 & Carabidae & 1,1 & Corylophidae & 1,1 \\
\hline Corylophidae & 1,8 & Corylophidae & 0,3 & Corylophidae & 0,8 & Corylophidae & 1,0 & Carabidae & 0,7 \\
\hline Latridiidae & 1,3 & Scydmaenidae & 0,3 & Latridiidae & 0,2 & Latridiidae & 0,6 & Scydmaenidae & 0,2 \\
\hline Total & 98,1 & & 98,7 & & 99,6 & & 99,0 & & 99,3 \\
\hline $70-80$ & \multirow{2}{*}{$\%$} & $80-90$ & \multirow{2}{*}{$\%$} & $90-100$ & \multirow{2}{*}{$\%$} & $100-110$ & \multirow{2}{*}{$\%$} & $110-120$ & \multirow{2}{*}{$\%$} \\
\hline Família & & Família & & Família & & Família & & Família & \\
\hline Nitidulidae & 36,4 & Nitidulidae & 48,7 & Nitidulidae & 45,2 & Nitidulidae & 55,9 & Nitidulidae & 52,0 \\
\hline Scarabaeidae & 35,6 & Curculionidae & 22,2 & Scarabaeidae & 24,7 & Scarabaeidae & 17,5 & Curculionidae & 27,3 \\
\hline Curculionidae & 14,3 & Scarabaeidae & 14,0 & Curculionidae & 16,3 & Curculionidae & 15,3 & Scarabaeidae & 12,0 \\
\hline Carabidae & 6,0 & Staphylinidae & 2,0 & Carabidae & 7,5 & Staphylinidae & 6,2 & Staphylinidae & 4,4 \\
\hline Staphylinidae & 4,0 & Corylophidae & 0,8 & Staphylinidae & 2,5 & Latridiidae & 1,7 & Carabidae & 3,2 \\
\hline Corylophidae & 0,7 & Carabidae & 0,5 & Latridiidae & 0,4 & Carabidae & 1,1 & Corylophidae & 0,2 \\
\hline Scydmaenidae & 0,5 & Scydmaenidae & 0,5 & Tenebrionidae & 0,6 & Corylophidae & 0,6 & Scydmaenidae & 0,2 \\
\hline Total & 97,5 & & 88,7 & & 97,2 & & 98,3 & & 99,3 \\
\hline
\end{tabular}


Considerando as duas subáreas separadamente, a subárea "A" apresentou uma abundância significativamente maior de coleópteros em relação a subárea "B". Na subárea "A" foram coletados $6.631(61 \%)$ espécimes, na subárea "B" 4.189 (39\%) espécimes (Tabela 3). Dentre as quatro famílias mais abundantes, a população de Nitidulidae foi superior nas duas áreas. Os resultados constatados neste trabalho são similares aos observados por Medri \& Lopes (2001), onde as famílias mais abundantes como Nitidulidae, Scarabaeidae e Staphylinidae eram as mais adaptadas ao ambiente antrópico.

Durante o período de coleta um total de 24 famílias de Coleoptera foram capturadas, valores semelhantes foram encontrados por Pinho (2003) no Pantanal de Poconé (MT) e Marinoni \& Ganho (2003b) no Parque Estadual de Vila Velha (PR).

A diversidade foi similar entre as áreas, sendo 1,22 na subárea "A" e 1,42 na subárea "B". Porém, a fauna de coleópteros foi diferente no número de indivíduos, conforme constatado pelo índice de Simpson $(0,37$ e 0,32$)$ para as subáreas "A" e "B" respectivamente.
A diversidade de famílias, ou até mesmo número de indivíduos por família, podem ter sido afetados pelo processo de fragmentação. A fragmentação tende a afetar as comunidades biológicas, seja pela abundância de indivíduos restritos a poucas famílias, como foi o caso de Nitidulidae, Curculionidae, Scarabaeidae e Staphylinidae ou pela captura de apenas um espécime por família: Aderidae, Anthicidae, Anthribidae, Cerambycidae, Chelonariidae, Endomychidae, Lampyridae, Meloidae e Silvanidae.

A ocorrência de famílias com grande número de indivíduos, conforme o constatado para Nitidulidae, Scarabaeidae e Staphylinidae por Medri \& Lopes em seu estudo, indica uma melhor adaptação destas famílias aos habitats fragmentados (Medri \& Lopes 2001). Este resultado é similar ao encontrado na Mata do Mergulhão, onde a maior abundância ocorreu na subárea "A". No entanto, fragmentos com elevado grau de impacto e/ou de tamanho reduzido estão sujeitos a menores índices de diversidade, principalmente por aumentar a abundância de espécies dependentes de um recurso em particular, por

Tabela 3. Abundância dos espécimes por família, em cada subárea "A" e "B" da Mata do Mergulhão, em Campos dos Goytacazes (RJ), capturadas em armadilha de solo, de agosto de 2003 a agosto de 2004.

Table 3. Abundance of specimens by family, in each sub-area "A" and "B" of the remnants of lowland Atlantic Forest, captured in pitfall trap, during August of 2003 to August of 2004.

\begin{tabular}{|c|c|c|c|c|c|}
\hline Famílias & Subárea A & Subárea B & Famílias & Subárea A & Subárea B \\
\hline Nitidulidae & 2912 & 1870 & Anobiidae & 8 & 3 \\
\hline Curculionidae & 2721 & 455 & Elateridae & 3 & 4 \\
\hline Scarabaeidae & 630 & 1389 & Mordellidae & 1 & 3 \\
\hline Staphylinidae & 122 & 204 & Meloidae & 1 & 0 \\
\hline Carabidae & 70 & 134 & Lampyridae & 1 & 0 \\
\hline Corylophidae & 65 & 41 & Aderidae & 1 & 0 \\
\hline Latrdiidae & 34 & 19 & Cerambycidae & 0 & 1 \\
\hline Scydmaenidae & 27 & 25 & Chelonariidae & 0 & 1 \\
\hline Hydrophilidae & 16 & 4 & Anthicidae & 0 & 1 \\
\hline Tenebrionidae & 7 & 12 & Silvanidae & 0 & 1 \\
\hline Chrysomelidae & 7 & 9 & Anthribidae & 0 & 1 \\
\hline Histeridae & 5 & 11 & Endomychidae & 0 & 1 \\
\hline Total (Subárea A): & 6631 & - & - & - & - \\
\hline Total (Subárea B): & 4189 & - & - & - & - \\
\hline
\end{tabular}

Tabela 4. Variação do número de famílias e indivíduos de Coleoptera capturados em armadilha de solo, durante o período de amostragem, agosto de 2003 à agosto de 2004 em função dos fatores abióticos (temperatura, precipitação e umidade relativa).

Table 4. Variation of the number of families and captured individuals of Coleoptera in pitfall trap, during August of 2003 to August of 2004, in function of the not biotics factors (temperature, precipitation and relative humidity).

\begin{tabular}{|c|c|c|c|c|c|}
\hline Período de coleta & Precipitação (mm) & T média ${ }^{\circ} \mathbf{C}$ & UR (\%) média & $\mathrm{N}^{\circ}$ de indivíduos & $\mathrm{N}^{0}$ de famílias \\
\hline Ago./2003 & 50,3 & 19,9 & 77,1 & 456 & 9 \\
\hline Set./2003 & 19,5 & 20,4 & 76,2 & 377 & 9 \\
\hline Out./2003 & 0,0 & 22,4 & 77,4 & 1198 & 16 \\
\hline Nov./2003 & 5,2 & 22,8 & 77,9 & 918 & 16 \\
\hline Dez./2003 & 135,8 & 25,0 & 81,9 & 1933 & 14 \\
\hline Jan./2004 & 146,0 & 24,5 & 83,5 & 754 & 14 \\
\hline Fev./2004 & 90,4 & 24,9 & 81,8 & 874 & 12 \\
\hline Mar./2004 & 12,3 & 24,5 & 80,6 & 1008 & 11 \\
\hline Abr./2004 & 99,8 & 23,9 & 84,0 & 827 & 11 \\
\hline Maio/2004 & 55,2 & 21,8 & 80,7 & 632 & 6 \\
\hline Jun./2004 & 32,1 & 20,2 & 81,2 & 836 & 10 \\
\hline Jul./2004 & 85,3 & 19,3 & 82,5 & 381 & 7 \\
\hline Ago./2004 & 15,0 & 20,3 & 78,8 & 626 & 8 \\
\hline
\end{tabular}


exemplo, troncos caídos, podem aumentar a população de espécies de decompositores (Schoereder et al. 2004).

Os resultados do levantamento da fauna de Coleoptera, na Mata do Mergulhão, indicaram abundância de coleópteros nos meses de temperaturas mais elevadas. Estes resultados são similares aos constatados por Marinoni \& Dutra (1991, 1996). Do total de indivíduos amostrados, 61,8\% (6.685 indivíduos) foram capturados no período chuvoso, outubro de 2003 a março de 2004, e 38,2\% (4.135 indivíduos) no período seco. Resultados similares foram encontrados por Lopes et al. (1994), Pinheiro et al. (1998) e Milhomem et al. (2003).

Foram constatadas maiores capturas nos meses de outubro e dezembro de 2003, e em março de 2004 (Tabela 4). Estes resultados são similares aos constatados por Diefenbach (1990) e Marinoni \& Ganho (2003a). Porém Oliveira et al. (2001), verificaram maiores quantidades de Coleoptera de setembro a janeiro.

De forma geral, pôde ser constatado nos levantamentos da fauna de Coleoptera de solo, que estes são mais abundantes no período de maiores temperaturas, e que a maioria das famílias de Coleoptera coletadas nas armadilhas de solo foi representada por poucos indivíduos.

As temperaturas médias registradas no período de coleta foram de $19,3{ }^{\circ} \mathrm{C}$, como mínima, em julho e de $25^{\circ} \mathrm{C}$ máxima, em dezembro, mostrando que a temperatura teve uma pequena variação durante o período de agosto de 2003 a agosto de 2004 (Tabela 4).

As armadilhas de coleta foram eficientes na captura dos espécimes da ordem Coleoptera de solo, na Mata do Mergulhão, remanescente de Mata Atlântica de baixada. O elevado número de indivíduos da família Nitidulidae na área " $A$ " indica que esta área é mais impactada. Os dados do levantamento podem ser utilizados como subsídios para novos estudos, enriquecimento das informações biológicas e avaliar o nível de perturbação neste ecossistema, que é de extrema importância para desenvolver programas de conservação, proteção, manejo e recuperação de sua biodiversidade.

\section{Agradecimentos}

Agradecemos ao Dr. Nelson Lamego por permitir a realização dos experimentos em sua fazenda, ao professor Dr. Gilberto Soares Albuquerque (Universidade Estadual do Norte Fluminense-UENF) pelo apoio e sugestões no trabalho, ao professor Dr. Alexandre Pio Viana (UENF) pelas análises estatísticas, ao Dr. Jatinder Singh Multani pelo auxílio na confecção do abstract. À Universidade Estadual do Norte Fluminense e a FAPERJ pelo financiamento do trabalho.

\section{Referências Bibliográficas}

CARLTON, C.E. \& ROBISON, H.W. 1998. Diversity of litter-dwelling beetles in the Ouachita highlans of Arkansas, USA (Insecta: Coleoptera). Biodivers. Conserv. 7(12):1589-1605.

CARVALHO, F.A., ALVARENGA, J.M.B., GOMES, J.M.L, SOUZA, J.S \& NASCIMENTO, M.T. 2006. Comunidade arbórea de uma floresta de baixada alluvial no município de Campos dos Goytacazes, RJ. Cerne Lavras 12(2):157-166.

CHUNG, A.Y.C., EGGLETON, P., SPEIGHT, M.R., HAMMOND, P.M. \& CHEY, V.K. 2000. The diversity of beetle assemblages in different habitat types in Sabah, Malaysia. Bull. Entomol. Res. 90(6):475-496.

DIDHAM, R.K., HAMMOND, P.M., LAWTON, J.H., EGGLETON, P. \& STORK, N.E. 1998a. Beetle species responses to tropical forest fragmentation. Ecol. Monogr. 68(3):295-323.

DIDHAM, R.K., HAMMOND, P.M., LAWTON. J.H., EGGLETON, P. \& STORK, N.E. 1998b. Trophic structure stability and extinction dynamics of beetles (Coleoptera) in tropical forest fragments. Philos. Trans. R. Soc. Lond. 353(3):437-451.

DIEFENBACH, L.M.G. 1990. Taxocenose de Carabideos (Insecta: Coleoptera: Carabidae) em um Parque Urbano (Porto Alegre, RS). Dissertação de
Mestrado, Universidade Federal do Rio Grande do Sul, Rio Grande do Sul, $192 \mathrm{p}$.

GANHO, N.G. \& MARINONI, R.C. 2003. Fauna de Coleoptera no Parque Estadual de Vila Velha, Ponta Grossa, Paraná, Brasil: abundância e riqueza das famílias capturadas através de armadilhas malaise. Rev. Bras. Zoo. 20(4):727-736.

LAWRENCE, J.F., HASTIGS, A.M., DALLWITZ, M.J., PAINE, T.A. \& ZURCHER, E.J. 1999. Beetles of the world. A Key and information system for families and subfamilies. CSIRO Publishing, Melbourne. (Version 1.0 MS-Windows CD-ROM).

LOPES, J., CONCHON, S., YUZAWA, S.K. \& KUHNLEIN, R.R.C. 1994 Entomofauna do Parque Estadual Mata dos Godoy: II. Scarabaeidae (Coleoptera) coletados em armadilhas de solo. Semina 15(2):121-127.

MAGURRAN, A.E. 1988. Ecological Diversity and its Measurement. Princeton University Press, Princeton, New Jersey, 179 p.

MARINONI, R.C. \& DUTRA, R.R.C. 1991. Levantamento da fauna entomológica no estado do Paraná. I. Introdução. Situações climática e florística de oito pontos de coleta. Dados faunísticos de agosto de 1986 a julho de 1987. Rev. Bras. Zoo. 8(1/2/3/4):31-73.

MARINONI, R.C. \& DUTRA, R.R.C. 1996. Levantamento da fauna entomológica no estado do Paraná II. Ctenuchidae (Lepidoptera). Rev Bras. Zoo. 13(2):435-461.

MARINONI, R.C. \& GANHO, N.G. 2003a. Sazonalidade de Nyssodrysina lignaria (Bates) (Coleoptera, Cerambycidae, Lamiinae), no Estado do Paraná, Brasil. Rev. Bras. Zoo. 20(1):141-152.

MARINONI, R.C. \& GANHO, N.G. 2003b. Fauna de Coleoptera no Parque Estadual de Vila Velha, Ponta Grossa, Paraná, Brasil. Abundância e riqueza das famílias capturadas através da armadilha de solo. Rev. Bras. Zoo. 20(4):737-744.

MEDRI, M.I. \& LOPES, J. 2001. Scarabaeidae (Coleoptera) do Parque Estadual Mata dos Godoy e de área de pastagem, no norte do Paraná, Brasil. Rev. Bras. Zoo. 18(1):135-141.

MILHOMEM, M.S., MELLO, F.Z.V. \& DINIZ, I.R. 2003. Técnicas de coleta de besouros copronecrófagos no Cerrado. Pesqu. Agropecu. Bras. 38(11):1-9.

MYERS, N., MITTERMEIER, R.A., MITTERMEIER, C.G., FONSECA, G.A.B. \& KENT, J. 2000. Biodiversity hotspots for conservation priorities. Nature, 403(24):853-858.

OLIVEIRA, H.G., ZANUNCIO, T.V., ZANUNCIO, J.C. \& SANTOS, G.P. 2001. Coleópteros associados a eucaliptocultura na região de Nova Era Minas Gerais, Brasil. Rev. Flor. Ambien. 8(1):52-60.

PETRONI, D.M. 2008. Diversidade de famílias de Coleoptera em diferentes fragmentos florestais no município de Londrina, PR. Dissertação de Mestrado, Universidade Estadual de Londrina, Londrina, 61 p.

PINHEIRO, F., DINIZ, I.R. \& KITAYAMA, K. 1998. Comunidade local de Coleoptera em cerrado: diversidade de espécies e tamanho do corpo. An. Soc. Entomol. Bras. 27(4):543-550

PINHO, N.G.C. 2003. Diversidade da artropodofauna em solo de Cambarazal no Pantanal de Poconé, Mato Grosso. Dissertação de Mestrado, Universidade Federal de Mato Grosso, Matro Grosso, $67 \mathrm{p}$

RODRIGUES, J.M.G. 1992. Abundância e distribuição vertical de coleópteros do solo em capoeira de Terra Firme, na região de Manaus-AM, Brasil. Acta Amazônica 22(3):323-333.

SCHOEREDER, J.H., SPERBER, C.F., SOBRINHO, T.G., RIBAS, C.R., GALBIATI, C., MADUREIRA, M.S. \& CAMPOS, R.B.F. 2004. Por que a riqueza de espécies de insetos é menor em fragmentos menores? Processos locais e regionais. Ecossistemas Brasileiros: Manejo e Conservação. Expressão Gráfica e Editora, Fortaleza, p. 31-36.

SCHUBART, H. \& BECK, L. 1968. Zur Coleopterenfauna amazonischer Böden. Amazoniana 1(4):311-322.

WINK, C., GUEDES, J.V.C., FAGUNDES, C.K. \& ROVEDDER, A.P. 2005. Insetos edáficos como indicadores da qualidade ambiental. Rev. Ciênc. Agrov. 4(1):60-75 